Article

\title{
Relationship between the Intake of n-3 Polyunsaturated Fatty Acids and Depressive Symptoms in Elderly Japanese People: Differences According to Sex and Weight Status
}

\author{
Hiromasa Tsujiguchi ${ }^{1, *}{ }^{1}$, Thao Thi Thu Nguyen ${ }^{1}\left(\mathbb{D}\right.$, Daisuke Goto ${ }^{1}$, Sakae Miyagi ${ }^{1}(\mathbb{D}$, \\ Yasuhiro Kambayashi ${ }^{1}$, Akinori Hara ${ }^{1}$, Yohei Yamada ${ }^{1}$, Haruki Nakamura ${ }^{1}$, Yukari Shimizu ${ }^{1}$, \\ Daisuke Hori ${ }^{2} \mathbb{D}$, Fumihiko Suzuki ${ }^{1}$, Koichiro Hayashi ${ }^{1}$, Satoko Tamai ${ }^{1}$ and \\ Hiroyuki Nakamura ${ }^{1}$ \\ 1 Department of Environmental and Preventive Medicine, Graduate School of Medical Science, \\ Kanazawa University, 13-1 Takaramachi, Kanazawa, Ishikawa 920-8640, Japan; toi_fs@yahoo.com (T.T.T.N.); \\ daigto211@gmail.com (D.G.); smiyagi@staff.kanazawa-u.ac.jp (S.M.); ykamba@med.kanazawa-u.ac.jp (Y.K.); \\ ahara@m-kanazawa.jp (A.H.); yamada503597@gmail.com (Y.Y.); haruki_nakamura@yahoo.co.jp (H.N.); \\ h_zu@me.com (Y.S.); fumi@dental.email.ne.jp (F.S.); k-hayashi@stu.kanazawa-u.ac.jp (K.H.); \\ stamai411@stu.kanazawa-u.ac.jp (S.T.); hiro-n@po.incl.ne.jp (H.N.) \\ 2 Occupational and Aerospace Psychiatry Group, Graduate School of Comprehensive Human Sciences, \\ University of Tsukuba, 1-1-1 Tennodai, Tsukuba, Ibaraki 305-8575, Japan; hori_d@mbr.nifty.com \\ * Correspondence: t-hiromasa@med.kanazawa-u.ac.jp; Tel.: +81-76-265-2288
}

Received: 19 March 2019; Accepted: 1 April 2019; Published: 3 April 2019

\begin{abstract}
PUFAs) have been shown to have preventive effects against depression. In this study, we aimed to investigate the associations between the intake of n-3 PUFAs and depression among people according to sex and weight status. We utilized cross-sectional data from the Shika study in Japan. The study was conducted between 2013 and 2016. Data were collected from adults older than 65 years. Invitation letters were distributed to 2677 individuals, 2470 of whom participated in the study $(92.3 \%)$. We assessed depressive states using the Japanese short version of the Geriatric Depression Scale (GDS-15). We assessed the intake of n-3 PUFAs using the validated food frequency questionnaire. One thousand six hundred thirty-three participants provided data, among which 327 (20.0\%) exhibited depressive symptoms. When we performed the stratified analysis by sex and weight status, there were significant inverse relationships between total n-3 PUFAs, individual n-3 PUFAs, and n-3/n-6 PUFAs ratio and depressive symptoms in overweight/obese females. No correlations were observed between n-3 PUFAs intake and depressive states in males. The results demonstrated a relationship between n-3 PUFAs deficiencies and depressive states, particularly in overweight/obese females. Dietary modifications may help to prevent depressive symptoms in overweight/obese females.
\end{abstract}

Keywords: n-3 PUFA; depressive; sex; weight status; Japanese

\section{Introduction}

Depression is one of the major illnesses in societies worldwide and affects personal well-being, the ability to work, and the use of healthcare resources [1]. The diagnosis of major depressive disorder (DSM-V) is given if an individual has experienced five or more of the identifying symptoms for a period of at least two weeks. Of these symptoms, at least one must be "a depressed mood" or "markedly diminished interest or pleasure". Other identifying symptoms include "significant weight loss", "insomnia or hypersomnia", 
"psychomotor agitation or retardation", "fatigue or loss of energy", "feelings of worthlessness or excessive or inappropriate guilt", "diminished ability to think or concentrate, or indecisiveness", and "recurrent thoughts of death, recurrent suicidal ideation without a specific plan, or a suicide attempt or a specific plan for committing suicide" [2]. An estimated 322 million individuals have depression worldwide [3]. A study on people aged 40 years or over in Japan reported $4.3 \%$ of males and $6.3 \%$ of females had severe depressive symptoms [4]. Depression is associated with significant disability [5]. Moreover, the economic cost of depression is a major issue for public health [6].

Depression occurs for various reasons and under different conditions. Nutrition, particularly polyunsaturated fatty acids (PUFAs), may influence depression. PUFAs are fatty acids that contain two or more unsaturated carbon-carbon double bonds with hydrogen atoms [7]. PUFAs are categorized as n-3 and n-6 fatty acids, based on their chemical structures. n-3 PUFAs include eicosapentaenoic acid (EPA), docosahexaenoic acid (DHA), docosapentaenoic acid (DPA), alpha-linolenic acid (ALA), and eicosatetraenoic acid (ETA) [7]. Longer-chain n-3 fatty acids (EPA and DHA) are synthesized by shorter-chain n-3 fatty acids (ALA) [7,8]. However, biological conversion is inefficient in humans [8,9]. In addition, shorter-chain fatty acids cannot be synthesized by humans [10,11]. Therefore, diet is an important source of these fatty acids [12].

n-3 PUFAs have been hypothesized to have preventive effects against depression. Previous studies have investigated the relationship between n-3 PUFAs dietary intake and depression. However, the findings obtained were conflicting [13]. Observational studies conducted on general populations are important from a prevention perspective.

Previous epidemiological studies have shown that the prevalence rate of depression is about twice in females compared to males [14]. In addition, a large number of epidemiological studies and meta-analyses have confirmed obesity to be associated with depression [15]. In recent decades, the consumption of n-3 PUFAs has been reduced and replaced by saturated fats from domestic animals and n-6 PUFAs from common vegetable oils and other sources, which changed dietary intakes of n-3 and n-6 PUFAs dramatically [16]. The higher prevalence rate of depression in females or obese people could be attributed to a vulnerability to n-3 PUFAs deficiency in these people. However, only a few researchers have evaluated the influences of sex or weight status on the relationship between n-3 PUFAs intake and depression. Understanding the influences of sex and weight status on the relationship between n-3 PUFAs intake and depression will be informative to address the target population and to maximize the beneficial effect so as to prevent depression.

Therefore, in this study, we aimed to investigate the associations between the intake of n-3 PUFAs and depression among people according to sex and weight status.

\section{Materials and Methods}

\subsection{Study Population}

We utilized cross-sectional data from the Shika study. The Shika study is an ongoing population-based survey that aims to develop advanced preventive methods for lifestyle-related diseases. It includes interviews, self-administered questionnaires, and comprehensive health examinations. Shika town is located in a rural area of the Ishikawa prefecture, Japan. The town has more than 20,000 residents [17]. Major industries include electronic component manufacturing, retail, and medical and welfare services [18]. The climate is humid subtropical. We selected four model districts in Shika town for the present study, which was conducted between October 2013 and December 2016. Data were collected from adults older than 65 years in the model districts. All adults older than 65 years who lived in these districts were eligible to participate in the present study. Invitation letters to participate were distributed to 2677 individuals, 2470 of whom participated in the study (92.3\%). All subjects gave their written informed consent for inclusion before they participated in the study. The study was conducted in accordance with the Declaration of Helsinki, and the protocol was approved by the Ethics Committee of Kanazawa University (No.1491). 


\subsection{Depressive States}

We assessed depressive states using the Japanese short version of the Geriatric Depression Scale (GDS-15), which consists of 15 questions developed for self-administrative surveys [19]. Higher scores indicate greater depressive symptoms [19]. A study that evaluated the validity of the Japanese version of GDS-15 recommended a cut-off score of 6/7 [20]. We used a cut-off point of 7, with scores $\geq 7$ indicating depressive symptoms. We included participants who answered more than 12 out of the 15 questions in the analysis.

\subsection{Nutrient Assessment}

We assessed the intake of n-3 PUFAs: total n-3 PUFAs, EPA+DHA, individual PUFAs (EPA, DHA, ALA, DPA, and ETA), n-3/n-6 PUFAs ratio, and n-6 PUFAs from the validated food frequency questionnaire. We used the brief-type self-administered dietary questionnaire (BDHQ) [21-23]. BDHQ is based on a comprehensive version of a validated self-administered questionnaire (i.e., DHQ) [24]. BDHQ asks about dietary history in the preceding month. BDHQ lists 58 food items $[22,23]$. The intake of n-3 PUFAs was estimated using a computer algorithm for BDHQ $[25,26]$. Participants who reported an energy intake per day of less than $600 \mathrm{kcal} /$ day (half of the required energy for the lowest physical activity category) or more than $4000 \mathrm{kcal} /$ day (1.5 times the energy intake required for the highest physical activity category) were excluded from the analyses because they were either extremely low or extremely high energy intakes [27]. The n-3 PUFAs intake was expressed as a percentage of the total energy intake.

\subsection{Other Variables}

The weight status was based on body mass index (BMI), calculated using self-reported heights and weights. BMI was computed based on the standard formula $\left(\mathrm{kg} / \mathrm{m}^{2}\right)$ [28]. It was used to classify participants as underweight $(<18.50)$, normal-weight $(18.50-24.99)$, overweight $(\geq 25.00)$, or obese $(\geq 30.00)$, based on the World Health Organization reference [28]. We categorized them as underweight/normal-weight $(<25.00)$ or overweight/obese $(\geq 25.00)$. Covariates included in the multivariable analysis consisted of age, energy intake (kcal/day), carbohydrate intake (\% energy), education (years of going to school), social activity (doing work or volunteering), living status (living alone or with someone), smoking status (current smoker, ex-smoker, or non-smoker), alcohol drinking, and history of chronic diseases (hypertension, stroke, myocardial infarction, diabetes, and hyperlipidemia). In the present study, participants who reported a history of stroke and/or myocardial infarction also had hypertension and/or diabetes. Therefore, we focused on hypertension, diabetes, and hyperlipidemia as confounders.

\subsection{Statistical Analysis}

Descriptive statistics were used to describe participant characteristics, depressive states, and n-3 PUFAs intake. Continuous variables were summarized as means and standard deviation (SD). Categorical variables were presented as numbers $(\mathrm{N})$ and percentages $(\%)$. Differences in characteristics, depressive states, and n-3 PUFAs intake between sexes were assessed using the Student's $t$-test (continuous variables) and the $\chi^{2}$ test (categorical variables). Differences in characteristics and n-3 PUFAs intake between participants with and without depressive symptoms were assessed using the Student's $t$-test and the $\chi^{2}$ test. Differences in the intakes of n-3 PUFAs among participants were also assessed using a two-way analysis of variance (two-way ANOVA) with depressive states and sex as the main factors. Both depressive states and sex were between factors. Tests for interactions of depressive states and sex were done to assess sex differences. We conducted post hoc tests in order to examine the differences in the n-3 PUFAs intakes between participants with and without depressive symptoms in each sex. The two-way ANOVA was also used with depressive states and weight status as the main factors. We also tested for interactions of depressive states and weight status. 
Post hoc tests in each weight status category were conducted. Participants were stratified by sex and weight status for multivariate logistic regression analyses. The dependent variable was the existence of depressive symptoms. The independent variables were the intakes of each n-3 PUFAs, the n-3/n-6 PUFAs ratio, and n-6 PUFAs. Multivariate logistic regression analyses were adjusted by age, energy intake, carbohydrate intake, education, social activity, living status, smoking status, alcohol drinking, and history of chronic diseases. The odds ratios (ORs) and 95\% confidence intervals (CIs) of each n-3 PUFAs for depressive states were calculated. A type I error of 0.05 was used for all analyses, with $P$ values between 0.05 and 0.10 considered as borderline significance. The Statistical Package for Social Science (SPSS) for MS Windows, version 23.0 (SPSS, Inc., New York, NY, USA) was used for statistical analyses.

\section{Results}

\subsection{Participant Characteristics}

The characteristics of the studied population are shown in Table 1. A total of 1633 participants provided data for the variables of interest and were included in the analyses. The number of males was 720, while that of females was 913. The average ages of the participants were $73.5(\mathrm{SD}=6.8)$ years for males and $75.2(\mathrm{SD}=7.8)$ years for females. A total of $327(20.0 \%)$ of participants had depressive symptoms. Among them, 147 (20.4\%) were male and 180 (19.7\%) were female. Females had significantly higher total n-3 PUFAs, ALA, and n-6 PUFAs intakes than males. The dietary sources of n-3 PUFAs in the studied population are shown in Table S1.

Table 1. Participant characteristics.

\begin{tabular}{|c|c|c|c|c|c|c|c|}
\hline & \multicolumn{2}{|c|}{ Total $(N=1633)$} & \multicolumn{2}{|c|}{ Male $(N=720)$} & \multicolumn{2}{|c|}{ Female $(N=913)$} & \multirow{2}{*}{$p^{*}$} \\
\hline & Ave $(n)$ & SD (\%) & Ave $(n)$ & SD (\%) & Ave $(n)$ & SD (\%) & \\
\hline Age & 74.5 & 7.4 & 73.5 & 6.8 & 75.2 & 7.8 & $<0.001$ \\
\hline BMI & 22.9 & 3.2 & 23.3 & 3 & 22.6 & 3.3 & $<0.001$ \\
\hline Energy (kcal) & 1824.1 & 610.9 & 2014.5 & 632.3 & 1673.9 & 549.1 & $<0.001$ \\
\hline Carbohydrate (\% energy) & 55.4 & 8.5 & 54.0 & 8.9 & 56.5 & 8.1 & $<0.001$ \\
\hline Education & 10.4 & 2.4 & 10.7 & 2.5 & 10.1 & 2.2 & $<0.001$ \\
\hline Social activity (none) & 1237 & $78.3 \%$ & 523 & $75.0 \%$ & 714 & $80.9 \%$ & 0.005 \\
\hline Household size (being single) & 207 & $12.9 \%$ & 56 & $7.9 \%$ & 151 & $16.9 \%$ & $<0.001$ \\
\hline Drinking & 905 & $58.5 \%$ & 526 & $75.4 \%$ & 379 & $44.6 \%$ & $<0.001$ \\
\hline Smoking & 165 & $10.4 \%$ & 146 & $20.7 \%$ & 19 & $2.1 \%$ & $<0.001$ \\
\hline Hypertension & 643 & $39.4 \%$ & 310 & $43.1 \%$ & 333 & $36.5 \%$ & 0.007 \\
\hline Diabetes & 229 & $14.0 \%$ & 122 & $16.9 \%$ & 107 & $11.7 \%$ & 0.003 \\
\hline Hyperlipidemia & 314 & $19.2 \%$ & 102 & $14.2 \%$ & 212 & $23.2 \%$ & $<0.001$ \\
\hline Depressive state & 327 & $20.0 \%$ & 147 & $20.4 \%$ & 180 & $19.7 \%$ & 0.725 \\
\hline Total n-3 PUFAs (\% energy) & 1.382 & 0.481 & 1.338 & 0.470 & 1.417 & 0.487 & 0.001 \\
\hline EPA+DHA (\% energy) & 0.539 & 0.315 & 0.531 & 0.310 & 0.545 & 0.320 & 0.368 \\
\hline EPA (\% energy) & 0.204 & 0.127 & 0.201 & 0.124 & 0.207 & 0.129 & 0.390 \\
\hline DHA (\% energy) & 0.335 & 0.189 & 0.330 & 0.186 & 0.339 & 0.192 & 0.354 \\
\hline DPA (\% energy) & 0.058 & 0.033 & 0.057 & 0.033 & 0.059 & 0.034 & 0.417 \\
\hline ALA (\% energy) & 0.714 & 0.224 & 0.679 & 0.211 & 0.741 & 0.230 & $<0.001$ \\
\hline ETA (\% energy) & 0.020 & 0.013 & 0.020 & 0.012 & 0.020 & 0.013 & 0.551 \\
\hline n-3/n-6 PUFAs ratio & 0.305 & 0.107 & 0.307 & 0.105 & 0.302 & 0.108 & 0.343 \\
\hline n-6 PUFAs & 5.149 & 1.339 & 4.928 & 1.265 & 5.323 & 1.370 & $<0.001$ \\
\hline
\end{tabular}

* $t$-tests for continuous variables and chi-square tests for categorical variables by sex ( $p$-values under 0.05 are highlighted in bold).

\subsection{Participant Characteristics and n-3 PUFAs Intake According to Depressive States}

Participant characteristics and n-3 PUFAs intake according to depressive states are shown in Table 2. Participants with depressive symptoms were significantly older than those without. Participants with depressive symptoms had a significantly lower energy intake, less education, less social activity, less household size, less alcohol drinking than those without. The levels of seven n-3 PUFAs were 
significantly lower in participants with depressive symptoms than in those without. Although no significant difference was observed between the two groups, the n-3/n-6 PUFAs ratio and the $n-6$ PUFAs were slightly lower in participants with depressive symptoms.

Table 2. Participant characteristics and n-3 polyunsaturated fatty acids (PUFAs) intake according to depressive states ( $t$-tests and chi-square tests).

\begin{tabular}{|c|c|c|c|c|c|}
\hline & \multicolumn{2}{|c|}{ Without Depressive States $(N=1306)$} & \multicolumn{2}{|c|}{ With Depressive States $(N=327)$} & $p^{*}$ \\
\hline Age & 73.8 & 7.1 & 77.3 & 8.1 & $<0.001$ \\
\hline Energy (kcal) & 1852.2 & 618.2 & 1712 & 568 & $<0.001$ \\
\hline Carbohydrate (\% energy) & 55.1 & 8.5 & 56.6 & 8.5 & 0.004 \\
\hline Household size (being single) & 159 & $12.4 \%$ & 48 & $15.0 \%$ & 0.206 \\
\hline Drinking & 746 & $60.3 \%$ & 159 & $51.1 \%$ & 0.003 \\
\hline Smoking & 138 & $10.8 \%$ & 27 & $8.7 \%$ & 0.264 \\
\hline Hypertension & 511 & $39.1 \%$ & 132 & $40.4 \%$ & 0.682 \\
\hline Diabetes & 180 & $13.8 \%$ & 49 & $15.0 \%$ & 0.576 \\
\hline DHA (\% energy) & 0.340 & 0.192 & 0.315 & 0.174 & 0.033 \\
\hline DPA (\% energy) & 0.059 & 0.034 & 0.055 & 0.030 & 0.032 \\
\hline ALA (\% energy) & 0.722 & 0.224 & 0.679 & 0.220 & 0.002 \\
\hline ETA (\% energy) & 0.021 & 0.013 & 0.019 & 0.011 & 0.028 \\
\hline n-3/n-6 PUFAs ratio & 0.307 & 0.109 & 0.295 & 0.100 & 0.082 \\
\hline n-6 PUFAs & 5.179 & 1.332 & 5.026 & 1.363 & 0.063 \\
\hline
\end{tabular}

$* t$-tests for continuous variables and chi-square tests for categorical variables by depressive states ( $p$-values under 0.05 are highlighted in bold).

\subsection{Influence of Sex on the Association between n-3 PUFAs Intake and Depressive States}

The influence of sex on the association between n-3 PUFAs intake and depressive states is shown in Table 3. There was a significant or borderline significant main effect of depressive states in seven $n-3$ PUFAs, excluding n-3/n-6 PUFAs ratio and n-6 PUFAs. The tests for interactions of sex and depressive states were statistically significant or tended to be significant in seven n-3 PUFAs and n-6 PUFAs, excluding n-3/n-6 PUFAs ratio, which indicated that the associations between n-3 PUFAs intake and depressive states differed by sex. Post hoc tests showed, among females, the levels of all seven n-3 PUFAs, n-3/n-6 PUFAs ratio, and n-6 PUFAs were significantly lower in participants with depressive states than in those without. In contrast, in males, none of the n-3 PUFAs, n-3/n-6 PUFAs ratio, or n-6 PUFAs levels were significantly lower in participants with depressive symptoms than in those without.

Table 3. Influence of sex on the association between n-3 PUFAs intake and depressive states (two-way ANOVA).

\begin{tabular}{|c|c|c|c|c|c|c|c|c|c|c|c|}
\hline & & \multicolumn{3}{|c|}{$\begin{array}{l}\text { Without Depressive States } \\
\qquad(N=1306)\end{array}$} & \multicolumn{3}{|c|}{$\begin{array}{l}\text { With Depressive States } \\
\qquad(N=327)\end{array}$} & \multirow{3}{*}{$p 1^{\mathrm{a}}$} & \multirow{3}{*}{$p 2^{\mathrm{b}}$} & \multirow{3}{*}{$p 3^{c}$} & \multirow{3}{*}{$p 4^{\mathrm{d}}$} \\
\hline & & \multirow{2}{*}{ Average } & \multicolumn{2}{|c|}{$95 \%$ CI } & \multirow{2}{*}{ Average } & \multicolumn{2}{|c|}{$95 \%$ CI } & & & & \\
\hline & & & Lower & Upper & & Lower & Upper & & & & \\
\hline \multirow[t]{2}{*}{ Total n-3 PUFAs } & Male $(N=720)$ & 1.340 & 1.301 & 1.379 & 1.329 & 1.254 & 1.403 & \multirow[b]{2}{*}{0.002} & \multirow[b]{2}{*}{0.008} & \multirow[b]{2}{*}{0.798} & \multirow[b]{2}{*}{$<0.001$} \\
\hline & Female $(N=914)$ & 1.450 & 1.415 & 1.486 & 1.281 & 1.217 & 1.346 & & & & \\
\hline \multirow[t]{2}{*}{$\mathrm{EPA}+\mathrm{DHA}$} & Male $(N=720)$ & 0.531 & 0.505 & 0.556 & 0.533 & 0.484 & 0.582 & \multirow[b]{2}{*}{0.045} & \multirow{2}{*}{0.034} & \multirow{2}{*}{0.937} & \multirow[b]{2}{*}{0.002} \\
\hline & Female $(N=914)$ & 0.561 & 0.537 & 0.585 & 0.480 & 0.440 & 0.521 & & & & \\
\hline \multirow[t]{2}{*}{ EPA } & Male $(N=720)$ & 0.201 & 0.191 & 0.211 & 0.201 & 0.181 & 0.221 & \multirow{2}{*}{0.030} & \multirow{2}{*}{0.030} & \multirow{2}{*}{0.995} & \multirow{2}{*}{$<0.001$} \\
\hline & Female $(N=914)$ & 0.213 & 0.204 & 0.223 & 0.179 & 0.163 & 0.196 & & & & \\
\hline \multirow[t]{2}{*}{ DHA } & Male $(N=720)$ & 0.329 & 0.314 & 0.345 & 0.332 & 0.302 & 0.361 & \multirow{2}{*}{0.058} & \multirow{2}{*}{0.037} & \multirow{2}{*}{0.898} & \multirow{2}{*}{0.003} \\
\hline & Female $(N=914)$ & 0.348 & 0.334 & 0.362 & 0.301 & 0.277 & 0.325 & & & & \\
\hline \multirow[t]{2}{*}{ DPA } & Male $(N=720)$ & 0.057 & 0.055 & 0.060 & 0.058 & 0.052 & 0.063 & \multirow{2}{*}{0.055} & \multirow{2}{*}{0.043} & \multirow[b]{2}{*}{0.944} & \multirow[b]{2}{*}{0.003} \\
\hline & Female $(N=914)$ & 0.060 & 0.058 & 0.063 & 0.052 & 0.048 & 0.056 & & & & \\
\hline
\end{tabular}


Table 3. Cont

\begin{tabular}{|c|c|c|c|c|c|c|c|c|c|c|c|}
\hline & & \multicolumn{3}{|c|}{$\begin{array}{l}\text { Without Depressive States } \\
\qquad(N=1306)\end{array}$} & \multicolumn{3}{|c|}{$\begin{array}{l}\text { With Depressive States } \\
\qquad(N=327)\end{array}$} & \multirow{3}{*}{$p 1^{\mathrm{a}}$} & \multirow{3}{*}{$p 2^{b}$} & \multirow{3}{*}{$p 3^{c}$} & \multirow{3}{*}{$p 4^{\mathrm{d}}$} \\
\hline & & \multirow{2}{*}{ Average } & \multicolumn{2}{|c|}{$95 \%$ CI } & \multirow{2}{*}{ Average } & \multicolumn{2}{|c|}{$95 \% \mathrm{CI}$} & & & & \\
\hline & & & Lower & Upper & & Lower & Upper & & & & \\
\hline \multirow[t]{2}{*}{ ALA } & Male $(N=720)$ & 0.681 & 0.664 & 0.699 & 0.668 & 0.633 & 0.704 & \multirow{2}{*}{0.004} & \multirow{2}{*}{0.047} & \multirow{2}{*}{0.528} & \multirow{2}{*}{$<0.001$} \\
\hline & Female $(N=914)$ & 0.754 & 0.738 & 0.771 & 0.687 & 0.654 & 0.720 & & & & \\
\hline \multirow[t]{2}{*}{ ETA } & Male $(N=720)$ & 0.020 & 0.019 & 0.021 & 0.020 & 0.018 & 0.022 & \multirow{2}{*}{0.047} & \multirow{2}{*}{0.053} & \multirow{2}{*}{0.976} & \multirow{2}{*}{0.003} \\
\hline & Female $(N=914)$ & 0.021 & 0.020 & 0.022 & 0.018 & 0.016 & 0.020 & & & & \\
\hline \multirow{2}{*}{$\begin{array}{l}\text { n-3/n-6 PUFAs } \\
\text { ratio }\end{array}$} & Male $(N=720)$ & 0.308 & 0.299 & 0.316 & 0.307 & 0.290 & 0.324 & \multirow{2}{*}{0.114} & \multirow{2}{*}{0.139} & \multirow{2}{*}{0.946} & \multirow{2}{*}{0.022} \\
\hline & Female $(N=914)$ & 0.306 & 0.298 & 0.314 & 0.286 & 0.272 & 0.300 & & & & \\
\hline \multirow[t]{2}{*}{ n-6 PUFAs } & Male $(N=720)$ & 4.927 & 4.819 & 5.036 & 4.933 & 4.719 & 5.147 & \multirow{2}{*}{0.102} & \multirow{2}{*}{0.089} & \multirow{2}{*}{0.964} & \multirow[b]{2}{*}{0.013} \\
\hline & Female $(N=914)$ & 5.377 & 5.281 & 5.473 & 5.102 & 4.908 & 5.295 & & & & \\
\hline
\end{tabular}

a: Between with and without depressive states groups. b: Interaction by depressive states groups and sex.

c: Bonferroni post hoc tests between males with and without depressive symptoms. ${ }^{\mathrm{d}}$ : Bonferroni post hoc tests between females with and without depressive symptoms. ${ }^{a-d}: p$-values under 0.05 are highlighted in bold.

\subsection{Influence of Weight Status on the Association between n-3 PUFAs Intake and Depressive States}

The influence of weight status on the association between n-3 PUFAs intake and depressive states is shown in Table 4. The main effects of depressive states were significant or tended to be significant in all n-3 PUFAs, the n-3/n-6 PUFAs ratio, and n-6 PUFAs. The interactions of weight status and depressive states tended to be statistically significant in EPA+DHA, EPA, DHA, and DPA. When we performed post hoc analyses among overweight/obese participants, the levels of seven n-3 PUFAs and the n-3/n-6 PUFAs ratio were significantly lower or tended to be significantly lower in participants with depressive symptoms than without depressive symptoms.

Table 4. Influence of weight status on the association between n-3 PUFAs intake and depressive states (two-way ANOVA).

\begin{tabular}{|c|c|c|c|c|c|c|c|c|c|c|c|}
\hline & & \multicolumn{3}{|c|}{$\begin{array}{l}\text { Without Depressive States } \\
\qquad(N=1306)\end{array}$} & \multicolumn{3}{|c|}{$\begin{array}{l}\text { With Depressive States } \\
(N=327)\end{array}$} & \multirow{3}{*}{$p 1^{\mathrm{a}}$} & \multirow{3}{*}{$p 2^{\mathrm{b}}$} & \multirow{3}{*}{$p 3^{\mathrm{cd}}$} & \multirow{3}{*}{$p 4^{\mathrm{d}}$} \\
\hline & & \multirow{2}{*}{ Average } & \multicolumn{2}{|c|}{$95 \% \mathrm{CI}$} & \multirow{2}{*}{ Average } & \multicolumn{2}{|c|}{$95 \% \mathrm{CI}$} & & & & \\
\hline & & & Lower & Upper & & Lower & Upper & & & & \\
\hline \multirow[t]{2}{*}{ Total n-3 PUFAs } & $\begin{array}{l}\text { Underweight/normal-weight } \\
\qquad(N=1255)\end{array}$ & 1.392 & 1.362 & 1.422 & 1.318 & 1.260 & 1.377 & $<0.001$ & 0.109 & 0.028 & 0.003 \\
\hline & $\begin{array}{c}\text { Overweight/obese } \\
(N=378)\end{array}$ & 1.434 & 1.380 & 1.487 & 1.244 & 1.131 & 1.357 & & & & \\
\hline $\mathrm{EPA}+\mathrm{DHA}$ & $\begin{array}{c}\text { Overweight/obese } \\
(N=378)\end{array}$ & 0.559 & 0.524 & 0.594 & 0.451 & 0.376 & 0.525 & & & & \\
\hline \multirow[t]{2}{*}{ EPA } & $\begin{array}{l}\text { Underweight/normal-weight } \\
\qquad(N=1255)\end{array}$ & 0.207 & 0.199 & 0.215 & 0.195 & 0.179 & 0.210 & 0.003 & 0.091 & 0.176 & 0.009 \\
\hline & $\begin{array}{c}\text { Overweight/obese } \\
(N=378)\end{array}$ & 0.212 & 0.198 & 0.227 & 0.168 & 0.138 & 0.198 & & & & \\
\hline DPA & $\begin{array}{c}\text { Overweight/obese } \\
(N=378)\end{array}$ & 0.060 & 0.056 & 0.064 & 0.049 & 0.041 & 0.057 & & & & \\
\hline \multirow[t]{2}{*}{ ALA } & $\begin{array}{l}\text { Underweight/normal-weight } \\
\qquad(N=1255)\end{array}$ & 0.717 & 0.703 & 0.731 & 0.677 & 0.649 & 0.704 & 0.005 & 0.669 & 0.010 & 0.067 \\
\hline & $\begin{array}{c}\text { Overweight/obese } \\
(N=378)\end{array}$ & 0.740 & 0.715 & 0.765 & 0.686 & 0.633 & 0.738 & & & & \\
\hline \multirow[t]{2}{*}{ ETA } & $\begin{array}{l}\text { Underweight/normal-weight } \\
\qquad(N=1255)\end{array}$ & 0.020 & 0.020 & 0.021 & 0.019 & 0.018 & 0.021 & 0.007 & 0.119 & 0.221 & 0.016 \\
\hline & $\begin{array}{c}\text { Overweight/obese } \\
(N=378)\end{array}$ & 0.021 & 0.020 & 0.022 & 0.017 & 0.014 & 0.020 & & & & \\
\hline $\begin{array}{l}n-3 / n-6 \text { PUFAs } \\
\text { ratio }\end{array}$ & $\begin{array}{l}\text { Underweight/normal-weight } \\
\qquad(N=1255)\end{array}$ & 0.307 & 0.300 & 0.313 & 0.300 & 0.287 & 0.313 & 0.030 & 0.201 & 0.007 & 0.014 \\
\hline
\end{tabular}


Table 4. Cont.

\begin{tabular}{|c|c|c|c|c|c|c|c|c|c|c|c|}
\hline & & \multicolumn{3}{|c|}{$\begin{array}{l}\text { Without Depressive States } \\
\qquad(N=1306)\end{array}$} & \multicolumn{3}{|c|}{$\begin{array}{l}\text { With Depressive States } \\
(N=327)\end{array}$} & \multirow{3}{*}{$p 1^{\mathrm{a}}$} & \multirow{3}{*}{$p 2^{\mathrm{b}}$} & \multirow{3}{*}{$p 3^{\mathrm{cd}}$} & \multirow{3}{*}{$p 4^{\mathrm{d}}$} \\
\hline & & \multirow{2}{*}{ Average } & \multicolumn{2}{|c|}{$95 \% \mathrm{CI}$} & \multirow{2}{*}{ Average } & \multicolumn{2}{|c|}{$95 \% \mathrm{CI}$} & & & & \\
\hline & & & Lower & Upper & & Lower & Upper & & & & \\
\hline \multirow[t]{2}{*}{ n-6 PUFAs } & $\begin{array}{l}\text { Underweight/normal-weight } \\
\qquad(N=1255)\end{array}$ & 5.148 & 5.065 & 5.232 & 5.018 & 4.854 & 5.181 & \multirow{2}{*}{0.079} & \multirow[t]{2}{*}{0.647} & \multirow[t]{2}{*}{0.162} & \multirow[t]{2}{*}{0.211} \\
\hline & $\begin{array}{l}\text { Overweight } \\
(N=378)\end{array}$ & 5.279 & 5.130 & 5.429 & 5.056 & 4.740 & 5.372 & & & & \\
\hline
\end{tabular}

${ }^{a}$ : Between with and without depressive states groups. ${ }^{b}$ : Interaction by depressive states and weight status.

c: Bonferroni post hoc tests between underweight/normal-weight participants with and without depressive symptoms.

$\mathrm{d}$ : Bonferroni post hoc tests between overweight/obese people with and without depressive symptoms. ${ }^{\text {a-d }}$ : $p$-values under 0.05 are highlighted in bold.

\subsection{Relationships between n-3 PUFAs Intake and Depressive States by Sex and Weight Status}

The relationships between n-3 PUFAs intake and depressive states by sex and weight status are shown in Table 5 and Figure 1. When we conducted stratified analyses by sex and weight status, there were significant inverse relationships for total n-3 PUFAs, EPA+DHA, EPA, DHA, DPA, ETA, and n-3/n-6 PUFAs ratio in overweight/obese females. The intakes of total n-3 PUFAs and ALA were also inversely related to depressive symptoms among underweight/normal-weight females. No correlations were observed between n-3 PUFAs intake or n-3/n-6 PUFAs ratio and depressive states in males.

Table 5. Relationship between n-3 PUFAs intake and depressive states by sex and weight status (logistic regression analysis).

\begin{tabular}{|c|c|c|c|c|c|c|c|c|c|}
\hline \multirow{3}{*}{ Weight Status } & \multirow{3}{*}{ PUFAs } & \multicolumn{4}{|c|}{ Male } & \multicolumn{4}{|c|}{ Female } \\
\hline & & \multirow{2}{*}{ OR } & \multicolumn{2}{|c|}{$95 \% \mathrm{CI}$} & \multirow{2}{*}{$p$} & \multirow{2}{*}{ OR } & \multicolumn{2}{|c|}{$95 \% \mathrm{CI}$} & \multirow{2}{*}{$p^{*}$} \\
\hline & & & Lower & Upper & & & Lower & Upper & \\
\hline \multirow{6}{*}{ Underweight/normal-weight } & $\mathrm{EPA}+\mathrm{DHA}$ & 1.000 & 1.000 & 1.001 & 0.362 & 1.000 & 0.999 & 1.000 & 0.407 \\
\hline & EPA & 1.001 & 0.999 & 1.003 & 0.383 & 0.999 & 0.997 & 1.001 & 0.330 \\
\hline & ALA & 1.000 & 0.998 & 1.001 & 0.639 & 0.998 & 0.997 & 1.000 & 0.013 \\
\hline & ETA & 1.011 & 0.988 & 1.034 & 0.364 & 0.994 & 0.975 & 1.014 & 0.550 \\
\hline & n-3/n-6 PUFAs ratio & 1.691 & 0.143 & 20.050 & 0.677 & 0.544 & 0.080 & 3.719 & 0.535 \\
\hline & n-6 PUFAs & 0.999 & 0.806 & 1.239 & 0.994 & 0.856 & 0.693 & 1.057 & 0.148 \\
\hline \multirow[t]{6}{*}{ Overweight/obesity } & Total n-3 PUFAs & 1.000 & 0.999 & 1.002 & 0.604 & 0.997 & 0.996 & 0.999 & 0.007 \\
\hline & $\mathrm{EPA}+\mathrm{DHA}$ & 1.000 & 0.999 & 1.002 & 0.557 & 0.997 & 0.994 & 0.999 & 0.007 \\
\hline & ALA & 1.000 & 0.998 & 1.002 & 0.955 & 1.000 & 0.997 & 1.003 & 0.783 \\
\hline & ETA & 1.009 & 0.970 & 1.050 & 0.640 & 0.922 & 0.866 & 0.982 & 0.012 \\
\hline & n-3/n-6 PUFAs ratio & 2.856 & 0.063 & 130.369 & 0.590 & 0.001 & 0.001 & 0.011 & 0.003 \\
\hline & n-6 PUFAs & 0.993 & 0.695 & 1.417 & 0.968 & 1.441 & 0.830 & 2.502 & 0.195 \\
\hline
\end{tabular}

* Adjusted for age, energy, carbohydrate, education, social activity, household size, drinking, smoking, hypertension, diabetes, and hyperlipidemia ( $p$-values under 0.05 are highlighted in bold). 
Male

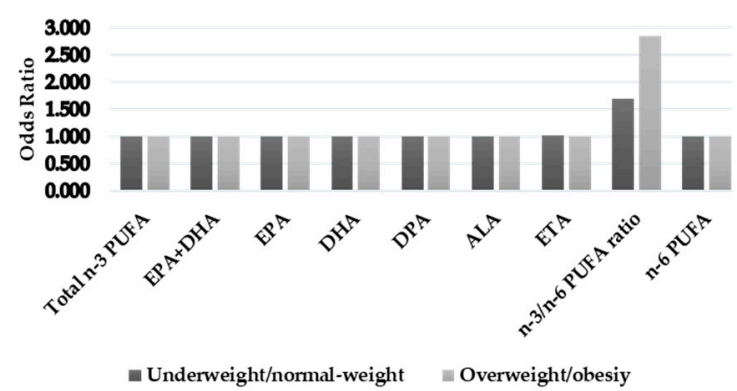

(a)
Female

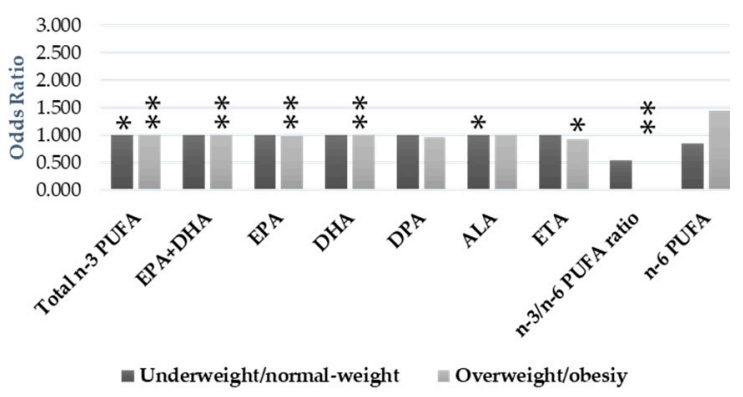

(b)

Figure 1. (a) Relationships between n-3 PUFAs intake and depressive states in males by weight status (* $p$-values under $0.05,{ }^{* *} p$-values under 0.01$)$, and (b) relationships between $n-3$ PUFAs intake and depressive states in females by weight status (* $p$-values under $0.05,{ }^{* *} p$-values under 0.01$)$.

\section{Discussion}

The results of the present study showed that a higher intake of n-3 PUFAs was associated with a lower risk of depressive symptoms, especially in overweight/obese females. To the best of our knowledge, this is the first study to demonstrate differences according to sex and weight status on the relationship between n-3 PUFAs intake and depressive symptoms.

The present results are consistent with previous findings, showing that n-3 PUFAs intake is inversely associated with depression [29]. According to a systematic review, most studies found that n-3 PUFAs intake was inversely associated with depression, though some studies found no association [29]. The difference observed between these findings may be due to the target population examined (middle-aged vs. elderly individuals) or the study design. In addition, most of the studies that investigated the relationship between n-3 PUFAs intake and depression were not stratified by sex or weight status. This may limit appropriate comparisons with the present results, which were stratified by sex and weight status.

Regarding participant characteristics, a relationship was found in females but not in males. This could be due to the difference in sample size between the males and females in our study ( $n=720$ (males) and $n=913$ (females)). Meanwhile, several epidemiological studies have found inverse associations between n-3 PUFAs and depressive symptoms in females but not in males [30-33]. Colangelo et al. [30] reported that higher total n-3 PUFAs, EPA+DHA, EPA, and DHA intakes predicted less severe depressive symptoms in females only. Beydoun et al. [31] also found that n-3 PUFAs intake and the n-3/n-6 PUFAs ratio were inversely associated with depressive symptoms in females. Persons et al. [33] found a positive association of dietary DHA+EPA with baseline depressive symptoms, though they found no associations at follow-up. In the study of Lucas et al. [33], although an inverse association of $\alpha$-linolenic acid with clinical depression was found, there was no association for total n-3 PUFAs. They also found that depression was positively linked to the n-3/n-6 PUFAs ratio.

Sex differences in the association between the intake of n-3 PUFAs and depressive symptoms can be explained by some mechanisms. Depression is considered to be secondary to inflammatory disorders. For example, inflammatory proteins, such as C-reactive protein (CRP) [34], and cytokines, such as interleukin (IL)-6 [35] and tumor necrosis factor (TNF)- $\alpha$ [36], have been elevated in people with depression. Pro-inflammatory cytokines alter serotonin metabolism, reduce synaptic plasticity, and increase the risk of developing depression [37-39]. The anti-inflammatory effects of n-3 PUFAs have been considered to play an important role in counteracting the inflammatory processes occurring in depression [40]. One possible explanation is that plasma DHA levels are higher in females than in males independent of DHA intake due to sex hormones [41,42]. It has been shown that estrogens may contribute to higher DHA concentrations [41,42]. 
It has not been clarified whether n-3 PUFAs intake is associated with depressive states among overweight/obese people. We could not find other epidemiological studies which focused on the modification by weight status on the association between n-3 PUFAs intake and depressive symptoms. It is suggested that obesity is also a disorder of inflammation [43]. Therefore, inflammation could link obesity with depression [44]. Hence, $n$-3 PUFAs could play a role in depressive symptoms via inflammatory processes, especially in the context of being overweight or obese.

Regarding ALA, a correlation was not observed in overweight/obese people. This could be due to a high n-6 PUFAs intake in overweight/obese people. Some studies suggest that overweight/obese people consume a diet with a low n-3/n-6 ratio [45]. It is reported that very little ALA is converted to EPA and DHA in the presence of high levels of n-6 PUFAs [46,47].

Furthermore, estrogen has been shown to increase inflammation $[48,49]$. Females have a stronger immune response than males $[49,50]$. The observed results in overweight/obese females could be due to a stronger immune response in females and the anti-inflammatory effects of $n-3$ PUFAs compared to males in all weight categories and underweight/normal-weight females.

A cross-national ecological study reported that an intake of $0.750 \mathrm{~g} /$ day of n-3 PUFAs was sufficient to protect $98 \%$ of the population from the risk of depression [51]. The World Health Organization (WHO) recommended an intake of $0.250 \mathrm{~g} /$ day of EPA plus DHA for non-pregnant/non-lactating adult females [52]. Dietary sources of longer-chain n-3 PUFAs are fish and wild animals (e.g., fatty fish, white fish, shellfish, other sea foods, eggs, and wild game) [11,53-55]. Dietary sources of shorter-chain n-3 PUFAs are plants (e.g., nuts, seeds, flaxseed, and rapeseed oil) [11,53-55].

Our study had several limitations. The study design was cross-sectional; therefore, the causality of relationships cannot be assessed. In addition, we obtained data from a subsample of a larger cohort. This may have introduced a selection bias, because these subsamples may not resemble the target population. Furthermore, despite being validated, the depression scale only suggests the presence of a depressive symptom. Another limitation is that self-reported assessment methods were subject to recall bias or misreporting. Overweight or obese individuals are more likely to misreport information [56]. However, the instruments used in the present study were validated, and evidence suggests that errors by misreporting may be cancelled out, at least in part, by energy adjustments $[57,58]$.

\section{Conclusions}

The results of the present study demonstrated a relationship between n-3 PUFAs deficiencies and depressive states, particularly in overweight/obese females. Our findings suggest that dietary modifications may help to prevent depressive symptoms in overweight/obese females, informing how interventions can effectively target specific populations for prevention. Further research is needed to clarify whether this relationship is consistent.

Supplementary Materials: The following are available online at http://www.mdpi.com/2072-6643/11/4/775/s1, Table S1: Dietary sources of the n-3 PUFAs intake.

Author Contributions: Conceptualization, H.N. (Hiroyuki Nakamura) and H.N. (Haruki Nakamura); methodology, H.N. (Hiroyuki Nakamura); formal analysis, H.T. and D.G.; investigation, H.T.; resources, H.N. (Hiroyuki Nakamura); data curation, H.T.; writing—original draft preparation, H.T. and T.T.T.N.; writing—review and editing, D.G., S.M., Y.K., A.H., Y.Y., H.N. (Haruki Nakamura), Y.S., D.H., F.S., K.H., S.T., and H.N. (Hiroyuki Nakamura); supervision, H.N. (Hiroyuki Nakamura); project administration, H.T.; funding acquisition, H.N. (Hiroyuki Nakamura).

Funding: This research was funded by the Establishment of Three Universities (Chiba/Kanazawa/Nagasaki) Graduate School of Innovative Preventive Medicine aiming for genuine disease prevention (2012-2017 Project to Promote National University Reform) from the Ministry of Education, Culture, Sports, Science, and Technology, Japan.

Acknowledgments: The Shika study was commissioned by Shika town. We would like to thank the study participants who responded to the survey and all of our colleagues at the Department of Environmental and Preventive Medicine, Graduate School of Medical Sciences, Kanazawa University.

Conflicts of Interest: The authors declare no conflict of interest. The funders had no role in the design of the study; in the collection, analyses, or interpretation of data; in the writing of the manuscript; or in the decision to publish the results. 


\section{References}

1. Wittchen, H.U.; Jacobi, F.; Rehm, J.; Gustavsson, A.; Svensson, M.; Jönsson, B.; Olesen, J.; Allgulander, C.; Alonso, J.; Faravelli, C.; et al. The size and burden of mental disorders and other disorders of the brain in Europe 2010. Eur. Neuropsychopharmacol. 2011, 21, 655-679. [CrossRef] [PubMed]

2. American Psychiatric Association. Diagnostic and Statistical Manual for Psychiatric Disorders, 5th ed.; American Psychiatric Association: Washington, DC, USA, 2013.

3. World Health Organization. Depression and Other Common Mental Disorders: Global Health Estimates; World Health Organization: Geneva, Switzerland, 2017; pp. 1-24.

4. Sekita, A.; Arima, H.; Ninomiya, T.; Ohara, T.; Doi, Y.; Hirakawa, Y.; Fukuhara, M.; Hata, J.; Yonemoto, K.; $\mathrm{Ga}, \mathrm{Y}$; , et al. Elevated depressive symptoms in metabolic syndrome in a general population of Japanese men: A cross-sectional study. BMC Public Health. 2013, 13, 862. [CrossRef] [PubMed]

5. Murray, C.J.; Lopez, A.D. Global mortality, disability, and the contribution of risk factors: Global Burden of Disease Study. Lancet 1997, 349, 1436-1442. [CrossRef]

6. Sobocki, P.; Jönsson, B.; Angst, J.; Rehnberg, C. Cost of depression in Europe. J. Ment. Health Policy Econ. 2006, 9, 87-98. [PubMed]

7. Grosso, G.; Galvano, F.; Marventano, S.; Malaguarnera, M.; Bucolo, C.; Drago, F.; Caraci, F. Omega-3 fatty acids and depression: Scientific evidence and biological mechanisms. Oxid. Med. Cell. Longev. 2014, 2014, 313570. [CrossRef]

8. Appleton, K.M.; Rogers, P.J.; Ness, A.R. Is there a role for n-3 long-chain polyunsaturated fatty acids in the regulation of mood and behaviour? A review of the evidence to date from epidemiological studies, clinical studies and intervention trials. Nutr. Res. Rev. 2008, 21, 13-41. [CrossRef]

9. Eaton, S.B.; Konner, M. Paleolithic nutrition. A consideration of its nature and current implications. N. Engl. J. Med. 1985, 312, 283-289. [CrossRef] [PubMed]

10. Haag, M. Essential Fatty Acids and the Brain. Can. J. Psychiatry-Revue Can. Psychiatr. 2003, 48, $195-203$. [CrossRef]

11. Ruxton, C.H.S.; Calder, P.C.; Reed, S.C.; Simpson, M.J.A. The impact of long-chain n-3 polyunsaturated fatty acids on human health. Nutr. Res. Rev. 2005, 18, 113. [CrossRef]

12. Ma, J.; Folsom, A.R.; Eckfeldt, J.H.; Lewis, L.; Chambless, L.E. Short- and long-term repeatability of fatty acid composition of human plasma phospholipids and cholesterol esters. The Atherosclerosis Risk in Communities (ARIC) Study Investigators. Am. J. Clin. Nutr. 1995, 62, 572-578. [CrossRef]

13. Giles, G.E.; Mahoney, C.R.; Kanarek, R.B. Omega-3 fatty acids influence mood in healthy and depressed individuals. Nutr. Rev. 2013, 71, 727-741. [CrossRef] [PubMed]

14. Piccinelli, M.; Wilkinson, G. Gender differences in depression. Critical review. Br. J. Psychiatry 2000, 177, 486-492. [CrossRef] [PubMed]

15. Mannan, M.; Mamun, A.; Doi, S.; Clavarino, A. Is there a bi-directional relationship between depression and obesity among adult men and women? Systematic review and bias-adjusted meta analysis. Asian J. Psychiatry 2016, 21, 51-66. [CrossRef]

16. Simopoulos, A.P. The importance of the ratio of omega-6/omega-3 essential fatty acids. Biomed. Pharmacother. 2002, 56, 365-379. [CrossRef]

17. Shika Town Shikatown Population. Available online: http://www.town.shika.ishikawa.jp/jyuumin/shika_ town_pop/shika_population.html (accessed on 1 March 2019).

18. Ishikawa Prefecture Ishikawa Statistical Information. Available online: http://toukei.pref.ishikawa.jp/search/ detail.asp?d_id=2489 (accessed on 1 March 2019).

19. Burke, W.J.; Roccaforte, W.H.; Wengel, S.P. The short form of the Geriatric Depression Scale: A comparison with the 30-item form. J. Geriatr. Psychiatry Neurol. 1991, 4, 173-178. [CrossRef]

20. Sugishita, K.; Sugishita, M.; Hemmi, I.; Asada, T.; Tanigawa, T. A Validity and Reliability Study of the Japanese Version of the Geriatric Depression Scale 15 (GDS-15-J). Clin. Gerontol. 2017, 40, 233-240. [CrossRef]

21. Okubo, H.; Sasaki, S.; Rafamantanantsoa, H.H.; Ishikawa-Takata, K.; Okazaki, H.; Tabata, I. Validation of self-reported energy intake by a self-administered diet history questionnaire using the doubly labeled water method in 140 Japanese adults. Eur. J. Clin. Nutr. 2008, 62, 1343-1350. [CrossRef] [PubMed] 
22. Kobayashi, S.; Murakami, K.; Sasaki, S.; Okubo, H.; Hirota, N.; Notsu, A.; Fukui, M.; Date, C. Comparison of relative validity of food group intakes estimated by comprehensive and brief-type self-administered diet history questionnaires against $16 \mathrm{~d}$ dietary records in Japanese adults. Public Health Nutr. 2011, 14, 1200-1211. [CrossRef] [PubMed]

23. Kobayashi, S.; Honda, S.; Murakami, K.; Sasaki, S.; Okubo, H.; Hirota, N.; Notsu, A.; Fukui, M.; Date, C. Both Comprehensive and Brief Self-Administered Diet History Questionnaires Satisfactorily Rank Nutrient Intakes in Japanese Adults. J. Epidemiol. 2012, 22, 151-159. [CrossRef]

24. Sasaki, S.; Yanagibori, R.; Amano, K. Self-administered diet history questionnaire developed for health education: A relative validation of the test-version by comparison with 3-day diet record in women. J. Epidemiol. 1998, 8, 203-215. [CrossRef]

25. Ministry of Health, Labor and Welfare of Japan. National Health and Nutrition Survey. Available online: http://ghdx.healthdata.org/record/japan-national-health-and-nutrition-survey-2013 (accessed on 1 March 2018).

26. Ministry of Education, Culture, Sports and Technology of Japan. Standard Tables of Food Composition in Japan 2015 Seventh Revised Version. Available online: http://www.mext.go.jp/en/policy/science_technology/ policy/title01/detail01/1374030.htm (accessed on 1 March 2019).

27. Murakami, K.; Sasaki, S.; Takahashi, Y.; Okubo, H.; Hosoi, Y.; Horiguchi, H.; Oguma, E.; Kayama, F. Dietary glycemic index and load in relation to metabolic risk factors in Japanese female farmers with traditional dietary habits. Am. J. Clin. Nutr. 2006, 83, 1161-1169. [CrossRef]

28. World Health Organization. BMI Classification. Available online: http://apps.who.int/bmi/index.jsp?introPage= intro_3.html (accessed on 1 March 2019).

29. Grosso, G.; Micek, A.; Marventano, S.; Castellano, S.; Mistretta, A.; Pajak, A.; Galvano, F. Dietary n-3 PUFA, fish consumption and depression: A systematic review and meta-analysis of observational studies. J. Affect. Disord. 2016, 205, 269-281. [CrossRef]

30. Colangelo, L.A.; He, K.; Whooley, M.A.; Daviglus, M.L.; Liu, K. Higher dietary intake of long-chain $\omega-3$ polyunsaturated fatty acids is inversely associated with depressive symptoms in women. Nutrition 2009, 25, 1011-1019. [CrossRef]

31. Beydoun, M.A.; Fanelli Kuczmarski, M.T.; Beydoun, H.A.; Hibbeln, J.R.; Evans, M.K.; Zonderman, A.B. w-3 Fatty Acid Intakes Are Inversely Related to Elevated Depressive Symptoms among United States Women. J. Nutr. 2013, 143, 1743-1752. [CrossRef]

32. Persons, J.E.; Robinson, J.G.; Ammann, E.M.; Coryell, W.H.; Espeland, M.A.; Harris, W.S.; Manson, J.E.; Fiedorowicz, J.G. Omega-3 fatty acid biomarkers and subsequent depressive symptoms. Int. J. Geriatr. Psychiatry 2014, 29, 747-757. [CrossRef]

33. Lucas, M.; Mirzaei, F.; Reilly, E.J.O.; Pan, A.; Willett, W.C.; Kawachi, I.; Koenen, K.; Ascherio, A. Dietary intake of n-3 and n-6 fatty acids and the risk of clinical depression in women: A 10-y prospective follow-up study 1-4. Am. J. Clin. Nutr. 2011, 93, 1337-1343. [CrossRef]

34. Häfner, S.; Baghai, T.C.; Eser, D.; Schüle, C.; Rupprecht, R.; Bondy, B.; Bedarida, G.; von Schacky, C. C-reactive protein is associated with polymorphisms of the angiotensin-converting enzyme gene in major depressed patients. J. Psychiatr. Res. 2008, 42, 163-165. [CrossRef]

35. Miller, G.E.; Stetler, C.A.; Carney, R.M.; Freedland, K.E.; Banks, W.A. Clinical depression and inflammatory risk markers for coronary heart disease. Am. J. Cardiol. 2002, 90, 1279-1283. [CrossRef]

36. Eller, T.; Vasar, V.; Shlik, J.; Maron, E. Pro-inflammatory cytokines and treatment response to escitaloprsam in major depressive disorder. Prog. Neuro-Psychopharmacol. Biol. Psychiatry 2008, 32, 445-450. [CrossRef]

37. Caraci, F.; Copani, A.; Nicoletti, F.; Drago, F. Depression and Alzheimer's disease: Neurobiological links and common pharmacological targets. Eur. J. Pharmacol. 2010, 626, 64-71. [CrossRef]

38. Huffman, J.C.; Celano, C.M.; Beach, S.R.; Motiwala, S.R.; Januzzi, J.L. Depression and cardiac disease: Epidemiology, mechanisms, and diagnosis. Cardiovasc. Psychiatry Neurol. 2013, 2013, 695925. [CrossRef] [PubMed]

39. Maes, M.; Yirmyia, R.; Noraberg, J.; Brene, S.; Hibbeln, J.; Perini, G.; Kubera, M.; Bob, P.; Lerer, B.; Maj, M. The inflammatory \& neurodegenerative (I\&ND) hypothesis of depression: Leads for future research and new drug developments in depression. Metab. Brain Dis. 2009, 24, 27-53. [CrossRef] [PubMed]

40. Parletta, N.; Milte, C.M.; Meyer, B.J. Nutritional modulation of cognitive function and mental health. J. Nutr. Biochem. 2013, 24, 725-743. [CrossRef] [PubMed] 
41. Giltay, E.J.; Gooren, L.J.G.; Toorians, A.W.F.T.; Katan, M.B.; Zock, P.L. Docosahexaenoic acid concentrations are higher in women than in men because of estrogenic effects. Am. J. Clin. Nutr. 2004, 80, 1167-1174. [CrossRef]

42. Bakewell, L.; Burdge, G.C.; Calder, P.C. Polyunsaturated fatty acid concentrations in young men and women consuming their habitual diets. Br. J. Nutr. 2006, 96, 93-99. [CrossRef] [PubMed]

43. Jantaratnotai, N.; Mosikanon, K.; Lee, Y.; McIntyre, R.S. The interface of depression and obesity. Obes. Res. Clin. Pract. 2017, 11, 1-10. [CrossRef]

44. Kurhe, Y.; Mahesh, R. Mechanisms linking depression co-morbid with obesity: An approach for serotonergic type 3 receptor antagonist as novel therapeutic intervention. Asian J. Psychiatry 2015, 17, 3-9. [CrossRef]

45. Manzel, A.; Muller, D.N.; Hafler, D.A.; Erdman, S.E.; Linker, R.A.; Kleinewietfeld, M. Role of "western diet" in inflammatory autoimmune diseases. Curr. Allergy Asthma Rep. 2014, 14, 404. [CrossRef]

46. Byelashov, O.A.; Sinclair, A.J.; Kaur, G. Dietary sources, current intakes, and nutritional role of omega-3 docosapentaenoic acid. Lipid Technol. 2015, 27, 79-82. [CrossRef] [PubMed]

47. Papanikolaou, Y.; Brooks, J.; Reider, C.; Fulgoni, V.L. U.S. adults are not meeting recommended levels for fish and omega-3 fatty acid intake: Results of an analysis using observational data from NHANES 2003-2008. Nutr. J. 2014, 13, 31. [CrossRef]

48. Cutolo, M.; Straub, R.H.; Bijlsma, J.W.J. Neuroendocrine-Immune interactions in synovitis. Nat. Clin. Pract. Rheumatol. 2007, 3, 627-634. [CrossRef]

49. Byrne, M.L.; O’Brien-Simpson, N.M.; Mitchell, S.A.; Allen, N.B. Adolescent-Onset Depression: Are Obesity and Inflammation Developmental Mechanisms or Outcomes? Child Psychiatry Hum. Dev. 2015, 46, 839-850. [CrossRef]

50. Klein, S.L. The effects of hormones on sex differences in infection: From genes to behavior. Neurosci. Biobehav. Rev. 2000, 24, 627-638. [CrossRef]

51. Hibbeln, J.R.; Nieminen, L.R.; Blasbalg, T.L.; Riggs, J.A.; Lands, W.E. Healthy intakes of n-3 and n-6 fatty acids: Estimations considering. Am. J. Clin. Nutr. 2006, 6, 1483S-1493S. [CrossRef]

52. World Health Organization; Uauy, R.E.A. Fats and Fatty Acids in Human Nutrition, Report of an Expert Consultation; Food and Agriculture Organization: Rome, Italy, 2008; Volume 91, ISBN 9789251067338.

53. British Nutrition Foundation. BNF Briefing Paper: $n-3$ Fatty Acids and Health; British Nutrition Foundation: London, UK, 1999; Volume 24, pp. 71-73.

54. Simopoulos, A.P. Evolutionary aspects of omega-3 fatty acids in the food supply. Prostaglandins. Leukot. Essent. Fatty Acids 2001, 60, 421-429. [CrossRef]

55. James, M.J.; Gibson, R.A.; Cleland, L.G. Dietary polyunsaturated fatty acids and inflammatory mediator production. Am. J. Clin. Nutr. 2000, 71,343S-348S. [CrossRef]

56. Livingstone, M.B.E.; Black, A.E. Markers of the validity of reported energy intake. J. Nutr. 2003, 133, 895S-920S. [CrossRef]

57. Lissner, L.; Troiano, R.P.; Midthune, D.; Heitmann, B.L.; Kipnis, V.; Subar, A.F.; Potischman, N. OPEN about obesity: Recovery biomarkers, dietary reporting errors and BMI. Int. J. Obes. 2007, 31, 956-961. [CrossRef] [PubMed]

58. Murakami, K.; Sasaki, S.; Takahashi, Y.; Uenishi, K.; Yamasaki, M.; Hayabuchi, H.; Goda, T.; Oka, J.; Baba, K.; Ohki, K.; et al. Misreporting of dietary energy, protein, potassium and sodium in relation to body mass index in young Japanese women. Eur. J. Clin. Nutr. 2008, 62, 111-118. [CrossRef]

(C) 2019 by the authors. Licensee MDPI, Basel, Switzerland. This article is an open access article distributed under the terms and conditions of the Creative Commons Attribution (CC BY) license (http://creativecommons.org/licenses/by/4.0/). 